\title{
Magnetic properties of Eu-Fe thin films
}

\author{
Katarzyna Balin, ${ }^{1,2, a)}$ Anna Nowak, ${ }^{1}$ Jacek Szade, ${ }^{1}$ Marcin Wojtyniak, ${ }^{1}$ \\ Dorota Wilgocka-Ślęzak, ${ }^{3}$ Michał Ślęzak, ${ }^{4}$ Tomasz Giela, ${ }^{3}$ Józef Korecki, ${ }^{3,4}$ \\ and Zbigniew Celinski ${ }^{2}$ \\ ${ }^{1}$ A. Chetkowski Institute of Physics, University of Silesia, 40-007 Katowice, Poland \\ ${ }^{2}$ Center for Magnetism and Magnetic Nanostructures, University of Colorado at Colorado Springs, \\ Colorado Springs, Colorado 80918, USA \\ ${ }^{3}$ Jerzy Haber Institute of Catalysis and Surface Chemistry, Polish Academy of Sciences, \\ 30-239 Kraków, Poland \\ ${ }^{4}$ Faculty of Physics and Applied Computer Science, AGH University of Science and Technology, \\ 30-059 Kraków, Poland
}

(Presented 15 January 2013; received 9 November 2012; accepted 21 December 2012; published online 3 April 2013)

\begin{abstract}
We studied the formation of alloys and compounds of the Eu-Fe binary system, in the form of thin films, prepared using molecular beam epitaxy. Diffraction and photoelectron spectroscopy investigations revealed mixing of the elements during the annealing at relatively low temperature. Magnetic studies showed ferromagnetic behavior up to $400 \mathrm{~K}$, and strong thermomagnetic effects and exchange bias indicating the presence of an antiferromagnetic phase and superparamagnetic particles. X-ray photoemission microscopy with dichroic contrast confirmed this hypothesis, showing two types of Eu-Fe alloys with various stoichiometries and showing ferromagnetically ordered particles of $100-500 \mathrm{~nm}$ in size. Ferromagnetic particles contain divalent Eu atoms, whereas the antiferromagnetic alloy is probably composed of the trivalent Eu atoms detected in the photoelectron spectra. (C) 2013 American Institute of Physics. [http://dx.doi.org/10.1063/1.4797622]
\end{abstract}

RareEarth $_{\mathrm{x}}$ TransitionMetal $_{\mathrm{y}}$ compounds with different stoichiometries have been extensively studied during the last decades, especially from the point of view of their interesting magnetic properties. ${ }^{1}$ In RE-Fe binary alloys, the magnetic ordering temperature is influenced mostly by the type of RE atoms present, and is proportional to the magnitude of $\mathrm{RE}$ spin $\left(\mathrm{T}_{\mathrm{C}}\right.$ is the highest for Gd-Fe with the largest RE spin, and it decreases for Tb-Fe, Dy-Fe alloys). ${ }^{1}$ The number of bulk form RE-Fe compounds is relatively large; nevertheless, it is limited to a few stoichiometries: $\mathrm{RE}_{2} \mathrm{Fe}_{3}, \mathrm{REFe}_{3}, \mathrm{RE}_{6} \mathrm{Fe}_{23}$, $\mathrm{RE}_{2} \mathrm{Fe}_{17 .}{ }^{2}$ There is only limited information about the formation of stable compounds between iron and europium. ${ }^{3}$

The magnetic properties of europium are directly connected to its valency state; $\mathrm{Eu}^{3+}$ is non-magnetic $(\mathrm{J}=0)$, while $\mathrm{Eu}^{2+}$ has a large pure spin moment $(\mathrm{J}=7 / 2)$. Metallic europium is divalent, but in alloys or intermetallic compounds, $\mathrm{Eu}$ may be in a divalent, trivalent, or intermediate-valency state. The ability to control the Eu valency, and consequently to switch between the non-magnetic and magnetic states of $\mathrm{Eu}$, led us to study the Eu-Fe system.

Similarly, as in the cases of Eu-(Mn, Cr), ${ }^{4,5}$ the $\mathrm{Eu}_{\mathrm{x}} \mathrm{Fe}_{\mathrm{y}}$ films were grown on $\mathrm{Si}$ or GaAs (with a $50 \mathrm{~nm}$ thick buffer layer of Mo) substrates in a wide $\mathrm{x}$ and $\mathrm{y}$ ranges (EuFe, $\mathrm{EuFe}_{3}, \mathrm{EuFe}_{5}, \mathrm{Eu}_{2} \mathrm{Fe}_{17}$ ) with a molecular beam epitaxy (MBE) deposition system. Two deposition modes-multilayer deposition and co-deposition were used. To form the $\mathrm{Eu}_{\mathrm{x}} \mathrm{Fe}_{\mathrm{y}}$ compounds, the films were annealed up to $500 \mathrm{~K}$. Most films were then capped by a protective thin film (up to $3 \mathrm{~nm}$ ) of Mo.

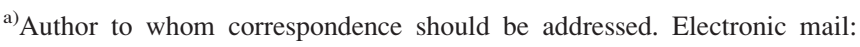
katarzyna.balin@us.edu.pl.
}

To examine the possibility of the formation of new ordered phases in the Eu-Fe system, we employed Reflection High Energy Electron Diffraction (RHEED) and X-ray Photoelectron Spectroscopy (XPS). Further structural characterization was carried out with the use of X-Ray Reflectivity (XRR) and X-Ray Diffraction (XRD). The magnetic properties were determined based on SQUID measurements and X-Ray Photoemission Microscopy (XPEEM).

Structural changes in the deposited layers were observed by RHEED, both after deposition and after the annealing process. In both cases, the films exhibit polycrystalline structure. Visible blurring of the rings indicates amorphization of the surface or some other dynamical processes. During the annealing process, observed changes in distances between diffused spots could indicate film texture changes, or that some additional strains occurred.

The X-ray reflectivity measurements (Fig. 1) showed that a uniform layer (see Fig. 1, inset), composed of a mixture of $\mathrm{Eu}$ and $\mathrm{Fe}$, is formed on the top of $\mathrm{Si} / \mathrm{Mo}$ substrate. Well-defined oscillations in the XRR patterns allowed for calculations (Reflectivitie 2000 program) of the thickness of the films. It was found that they are in good agreement with the assumed ones; typically the calculated thicknesses were several angstroms smaller than the assumed ones. This would suggest that the $\mathrm{Eu}$ and $\mathrm{Fe}$ atoms are mixing, and form more closely packed structures in such mixtures.

Possible formation of new $\mathrm{Eu}_{\mathrm{x}} \mathrm{Fe}_{\mathrm{y}}$ phases has been verified through the XRD research. Unfortunately, no atomically ordered intermetallic phase could be resolved. Even in the low incident angle analysis mode, the only apparent diffraction peaks come from the substrate or the protective Mo coating. 


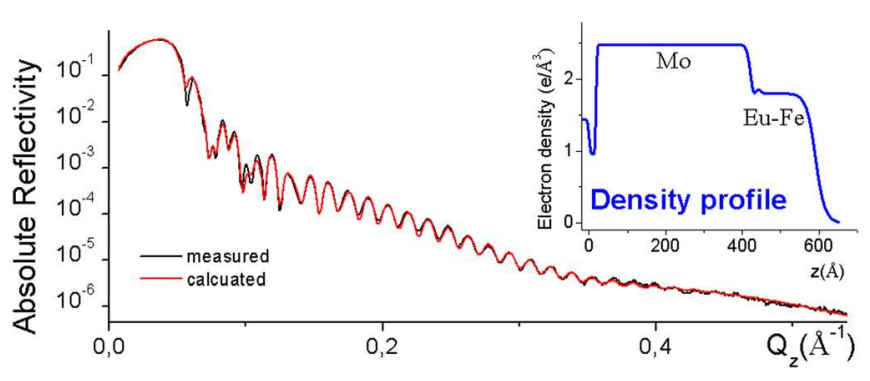

FIG. 1. Electron density profile (blue) calculated from the specular reflectivity (black and red) for the film with assumed stoichiometry $\mathrm{EuFe}_{5}$.

The photoemission spectra of core levels were systematically taken after the deposition of Eu-Fe multilayers or codeposited layer and later during the annealing process. For all examined films in the Eu-Fe system, both Eu and Fe exhibit typical metallic behavior after deposition. Chemical shifts of the core levels (e.g., Eu $3 d$ up to $8.9 \mathrm{eV}$ and $\mathrm{Fe} 2 p$ up to $0.2 \mathrm{eV}$ ) and relative changes in the $\mathrm{Eu} 4 d / \mathrm{Fe} 2 p$ line area ratio monitored by the XPS during the annealing process indicate mixing between Eu and Fe layers. A relatively low content of oxygen was observed after the annealing process. For almost all examined films of the Eu-Fe system, valence transition from $\mathrm{Eu}^{2+}$ to $\mathrm{Eu}^{3+}$ was observed (see Fig. 2), however, for selected compositions, the $\mathrm{Eu}^{2+}$ was still observed by the low intensity contribution to the $3 d$ and $4 d$ spectra.

Magnetic properties of the annealed films were studied with the use of a SQUID magnetometer in the temperature range of $1.9-400 \mathrm{~K}$ and in fields up to $7 \mathrm{~T}$. All the measured samples showed similar features (see Fig. 3)-ferromagnetic behavior up to the highest available temperature of $400 \mathrm{~K}$ and strong thermomagnetic effects. Typical for Eu-Fe system temperature dependence of magnetic moment (M-T), obtained in both zero-field cooled (ZFC) and field cooled (FC) modes, are different in nature than that observed in EuMn or Eu-Cr systems. ${ }^{4,5}$ Here, the magnetic behavior is associated mainly with the iron atoms or their clusters. From magnetization measurements obtained with the use of Physical Property Measurement System (PPMS magnetometer), we estimate $T_{c}$ to be at least $600 \mathrm{~K}$. For higher temperatures, irreversible degradation of the films was observed.

The most important feature of the M-T curves obtained at applied field of $100 \mathrm{Oe}$ is a strong thermomagnetic effect seen as irreversibility in FC and ZFC modes. The irreversibility can be attributed to the slowing of the spin relaxation times, which is caused by the distribution of the coercivity in

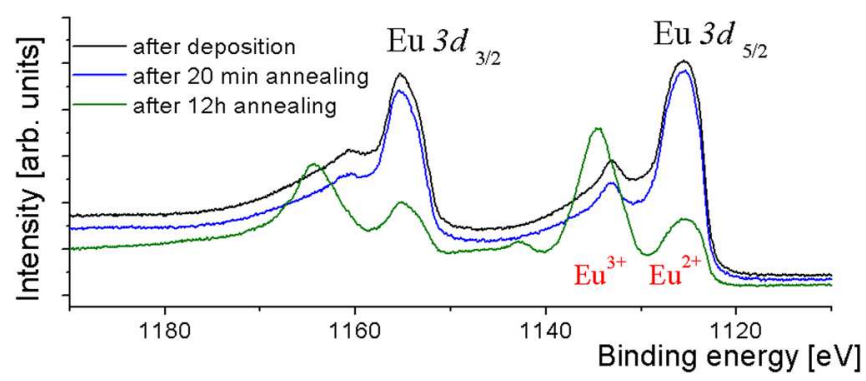

FIG. 2. Changes of Eu $3 d$ core level during the annealing process for the $\mathrm{EuFe}_{5}$ film.

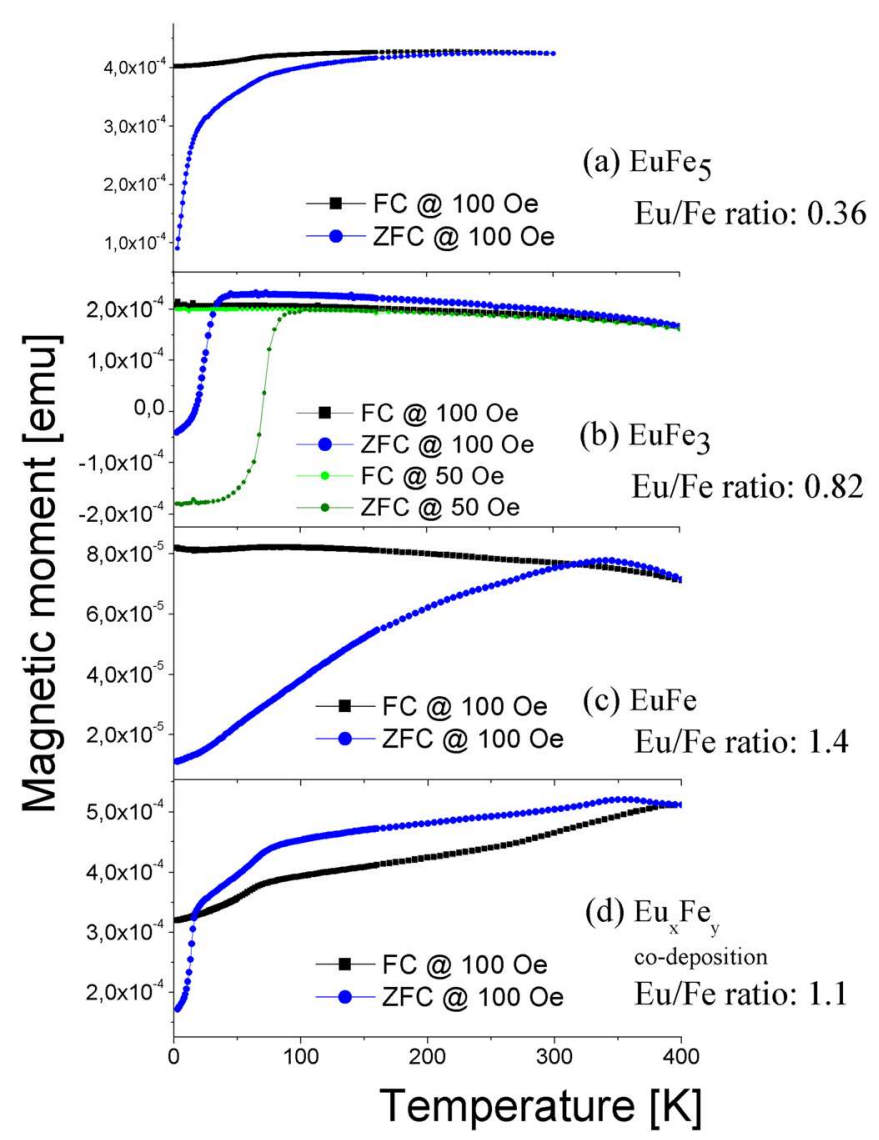

FIG. 3. M-T curves obtained for films with assumed stoichiometries (a) $\mathrm{EuFe}_{5}$, (b) $\mathrm{EuFe}_{3}$, (c) EuFe, (d) $\mathrm{Eu}_{\mathrm{x}} \mathrm{Fe}_{\mathrm{y}}$. Listed Eu/Fe ratios have been calculated from the XPS spectra obtained after the annealing process.

ferromagnetic particles. For the M-T curves obtained in measurements performed in ZFC mode, the magnetic moment increases more rapidly than for those performed in FC mode. This behavior is a typical characteristic of the blocking process for an assembly of superparamagnetic nanoparticles, but can be also linked to the spin glass materials. ${ }^{6,7}$ Frustration in the magnetic ordering can also lead for similar thermomagnetic effects.

Besides the irreversibility, there are a number of additional important features manifested in the M-T curves in both modes. One characteristic feature, which can be seen in M-T curves obtained in ZFC mode, is the presence of a broad maximum. The maxima in the M-T curves measured in the ZFC mode are related to the superparamagnetic unblocking process of single domain particles (in other words, it is related to the average blocking temperature of the particles). Most likely, the particles become superparamagnetic at temperatures above that observed maximum.

Looking more closely at M-T curves measured in FC mode, some additional features allow us to qualify two of the films as spin glass or super spin glass like materials. The films with the $\mathrm{Eu} / \mathrm{Fe}$ ratio 0.82 and 1.4 have minima in the M-T curves in FC mode at $18 \mathrm{~K}$ and $22.3 \mathrm{~K}$, respectively. This behavior is only observed in spin glass and super spin glass systems. ${ }^{6,7}$

Another important feature is an unusual and rarely observed effect; the value of the magnetic moment measured in ZFC mode exceeds the one found in the FC mode. This 
effect was observed for three films (see Figs. 3(b)-3(d)). Such an unusual effect was seen only in a very limited number of materials, for example, in amorphous flash-evaporated Pddoped NiMn thin films. ${ }^{8}$ With regard to the results presented in Fig. 3, the model proposed in Refs. 8 and 9 could be introduced with the assumption that a layer of pure $\mathrm{Fe}$ or $\mathrm{Eu}-\mathrm{Fe}$ mixture with some specified $\mathrm{Eu} / \mathrm{Fe}$ ratio plays the role of the classical ferromagnetic phase, whereas the antiferromagnetic regions consist of a Eu-Fe mixture (with different $\mathrm{Eu} / \mathrm{Fe}$ ratio) or pure Eu. The XRR results, however, show no separated Eu, $\mathrm{Fe}$, or Eu-Fe layers, therefore, we attribute each particular region to relatively small (too small to give peaks in XRD diffraction pattern) $\mathrm{Eu}$ or Eu-Fe nanoparticles. This effect was the greatest for the co-deposited film (Fig. 3(d)), which might be an additional indication that the antiferromagnetic phase is rather uniformly distributed in the film.

The observed field dependence of the peak in M-T curves (Fig. 3(b)) in the ZFC mode as well as rather weak changes in the temperature dependence of the FC magnetization indicate a cluster spin glass like behavior, with some strong interactions between the particles. ${ }^{10}$ The separation of the FC and ZFC branches of the M-T curves obtained at $50 \mathrm{Oe}$ and $100 \mathrm{Oe}$ for the $\mathrm{EuFe}_{3}$ film is still visible (Fig. 3(b)). The blocking temperature decreases as the field increases from 50 to 100 Oe. This essentially means that for higher applied fields, the effective spin reorientation barriers are lowered. Additionally, for measurements performed in the applied field of $50 \mathrm{Oe}$, in contrast to the one performed at $100 \mathrm{Oe}$, the M-T curves in ZFC mode are not above the one measured in FC mode. This would suggest that a $50 \mathrm{Oe}$ external field is too low to see the impact of antiferromagnetically ordered regions on the FC and ZFC branches, as was observed for $100 \mathrm{Oe}$.

For all examined films, the hysteresis loops revealed a decreasing value of coercive field with increasing temperature. The exchange bias phenomenon was observed in the $\mathrm{M}-\mathrm{H}$ hysteresis loops for all examined stoichiometries and for both deposition modes: multilayer deposition and codeposition process. Exchange biasing in the uniform layer confirms the presence of antiferromagnetically ordered regions. We attribute the exchange biasing to the interaction between anti-ferromagnetically oriented regions originating from the Eu-Fe alloy, and a ferromagnetic $\mathrm{Fe}$ or $\mathrm{Eu}-\mathrm{Fe}$ phase. Similarly, exchange biasing was observed in ferromagnetic Co layers with embedded antiferromagnetic $\mathrm{CoO}$ nanoparticles. ${ }^{11}$ To confirm the origin of the exchange biasing, we studied the EuFe ${ }_{5}$ film with the use of the XPEEM microscope at the PolLux beamline of the Swiss Light Source (SLS) synchrotron. The ELMITEC (PEEMIII) spectromicroscope was used, and measurements were performed at the room temperature. The photoemission images were registered for photon energies corresponding to the $\mathrm{Fe} \mathrm{L}_{2,3}$ edge and $\mathrm{Eu}_{4,5}$ edge for right and left circularly polarized light. The photoemission maps exhibit a clear heterogeneity of the film. There are regions $(100-500 \mathrm{~nm}$ in size) rich in Eu embedded in the $\mathrm{Eu}_{\mathrm{x}} \mathrm{Fe}_{\mathrm{y}}$ matrix (Fig. 4). These regions show a clear dichroic effect obtained by subtraction of the RCP and LCP intensity maps obtained at the Eu $\mathrm{M}_{4,5}$ edge, while the Fe L lines hardly show any dichroism. This may be

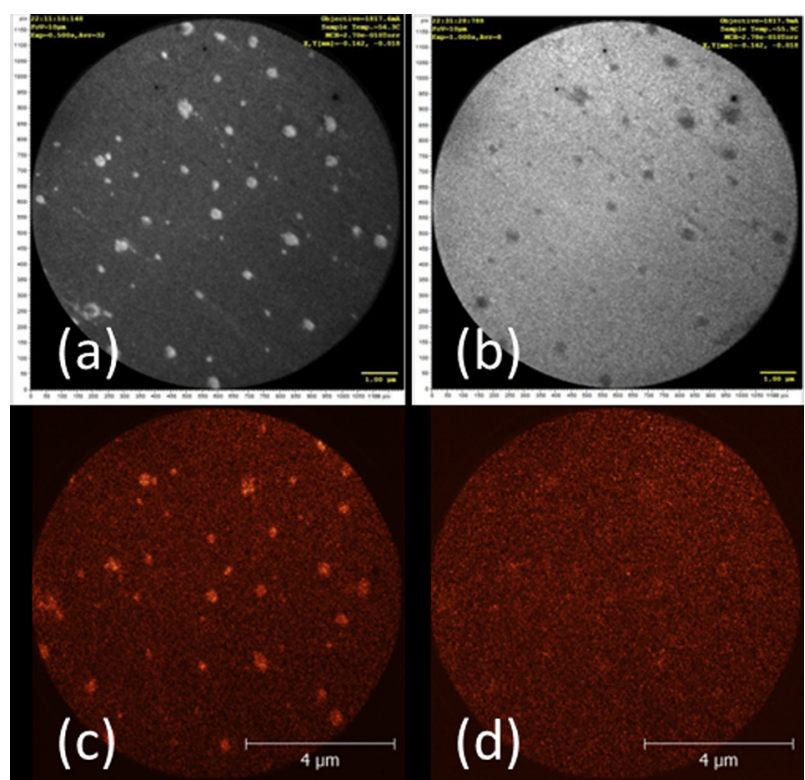

FIG. 4. PEEM images of $\mathrm{EuFe}_{0.36}$ film obtained at the photon energies of Eu $\mathrm{M}_{4,5}$ (a) and $\mathrm{Fe} \mathrm{L}_{2,3}$ (b) absorption edges and magnetic dichroism in photoemission (XPEEM images) obtained by subtracting the images acquired with left (LCP) and right (RCP) circularly polarized light energies corresponding to the $\mathrm{Eu} \mathrm{M}_{4,5}$, (c) field of view- $10 \mu \mathrm{m}$.

related to strong $\mathrm{Fe}$-Eu interactions, leading to ferromagnetic ordering within the Eu rich spots. These spots contain magnetic $\mathrm{Eu}^{2+}$ atoms, whereas the matrix is probably rich in non-magnetic $\mathrm{Eu}^{3+}$ atoms. The lack of dichroism in the $\mathrm{Fe}$ rich matrix means that this region is ordered antiferromagnetically. Taken together, this would explain the exchange biasing and freezing of the ferromagnetic particles observed for the ZFC mode. The ferromagnetic phase may have a well-defined $\mathrm{Eu} / \mathrm{Fe}$ ratio, though it is not clear whether any atomically ordered phase is formed.

Even if the formation of new ordered $\mathrm{Eu}-\mathrm{Fe}$ compounds was not observed, the Eu-Fe films still exhibit a few interesting properties. The RHEED, XRR, as well as XPS measurements indicate mixing between $\mathrm{Eu}$ and $\mathrm{Fe}$ layers. The valence transition of europium from $\mathrm{Eu}^{2+}$ to $\mathrm{Eu}^{3+}$ was observed for most samples. The magnetic properties, verified via SQUID, PPMS, and XPEEM, result from the presence of an antiferromagnetic phase and superparamagnetic regions composed from a mixture of $\mathrm{Eu}$ and $\mathrm{Fe}$ atoms with different $\mathrm{Eu} / \mathrm{Fe}$ ratios.

This work was supported by the NSF Grant No. DMR0907053 and by the SPINLAB project financed by the EU European Regional Development Fund.

${ }^{1}$ H. R. Kirchmayr et al., J. Magn. Magn. Mater. 8, 1-42 (1978).

${ }^{2}$ B. Predel, in Phase Equilibria, Crystallographic and Thermodynamic Data of Binary Alloys (Landolt-Börnstein, New Series, Vol. IV/5e), edited by O. Madelung (Springer, Berlin - Heidelberg, 1995).

${ }^{3}$ N. Yuan-Tao et al., J. Less-Common Met. 147, 167-173 (1989).

${ }^{4}$ K. Balin et al., J. Appl. Phys. 107, 09E154 (2010).

${ }^{5}$ K. Balin et al., J. Appl. Phys. 109, 07E323 (2011).

${ }^{6}$ S. Bedanta et al., J. Phys. D: Appl. Phys. 42, 013001 (2009).

${ }^{7}$ M. Sasaki et al., Phys. Rev. B 71, 104405 (2005).

${ }^{8}$ Y. Oner et al., J. Appl. Phys. 89, 7044 (2001).

${ }^{9}$ Y. Imry and S.-K. Ma, Phys. Rev. Lett. 35, 1399 (1975).

${ }^{10}$ D. Fiorani et al., J. Magn. Magn. Mater. 196-197, 143 (1999).

${ }^{11}$ J. Nogués et al., J. Magn. Magn. Mater. 192, 203 (1999). 